\title{
Lipid Metabolism, Metabolic Syndrome, and Cancer
}

Fang Hu, Yingtong Zhang and Yuanda Song

Additional information is available at the end of the chapter

http://dx.doi.org/10.5772/51821

\section{Introduction}

Metabolism is the process of making energy and cellular molecules from breaking down the food that made up of proteins, carbohydrates and fats etc. A metabolic disorder occurs when abnormal chemical reactions disrupt this process. When this happens, our body might have too much of some substances or too little of other ones that we need to stay healthy. Metabolic syndrome, a combination of several metabolic risk factors including abdominal obesity, insulin resistance, hypertension, and atherogenic dyslipidemia, is one of the most common health problems in the modern society. Increasingly accumulated evidence from epidemiologic and basic research data, as well as translational, clinical, and intervention studies suggested that metabolic syndrome may be an important etiologic factor for the onset of cancer. In fact cancer has long been indicated as a metabolic disease due to aberrant energy metabolism caused by mitochondrial damage.

In living cells, processes of carbohydrate metabolism, lipid metabolism and energy metabolism are closely related. Metabolic syndrome (MS), such as diabetes, obesity, hyperlipidimia, and hypertension, is, more or less, associated with abnormal lipid metabolism. As a metabolic disease, cancer is caused by impaired energy metabolism due to impaired mitochondrial function, which is linked with abnormal mitochondrial membrane lipids, especially cardiolipin content [1]. Recent studies have indicated that abnormalities in cellular lipid metabolism are involved in both pathogenesis of metabolic syndrome and various cancers $[2,3]$.

As the major component of membranes and energy resources, cellular lipids, including phospholipids and neutral lipids (mainly triacylglycerols and sterol esters), play a crucial role for both cellular and physiological energy homeostasis. As cellular membrane structure components, phospholipids are important for cellular membrane remodeling and cellular 
proliferation. Disruption of phospholipid homeostasis may lead to carcinogenesis and MS $[4,5]$. Triacylglycerol, as an important energy storage form, is closely related to glucose homeostasis and its disregulation is associated with onset of MS such as diabetes, obesity, and cardiovascular diseases [6].

In this chapter, we focused on the metabolism of phospholipid and triacylglycerol (including fatty acids) and discussed the association of lipid metabolism disorders with pathogenesis of MS and cancer as shown in Figure1. We also discussed the emerging role of omega-3 polyunsaturated fatty acids in preventing lipid disorder associated MS and cancer.

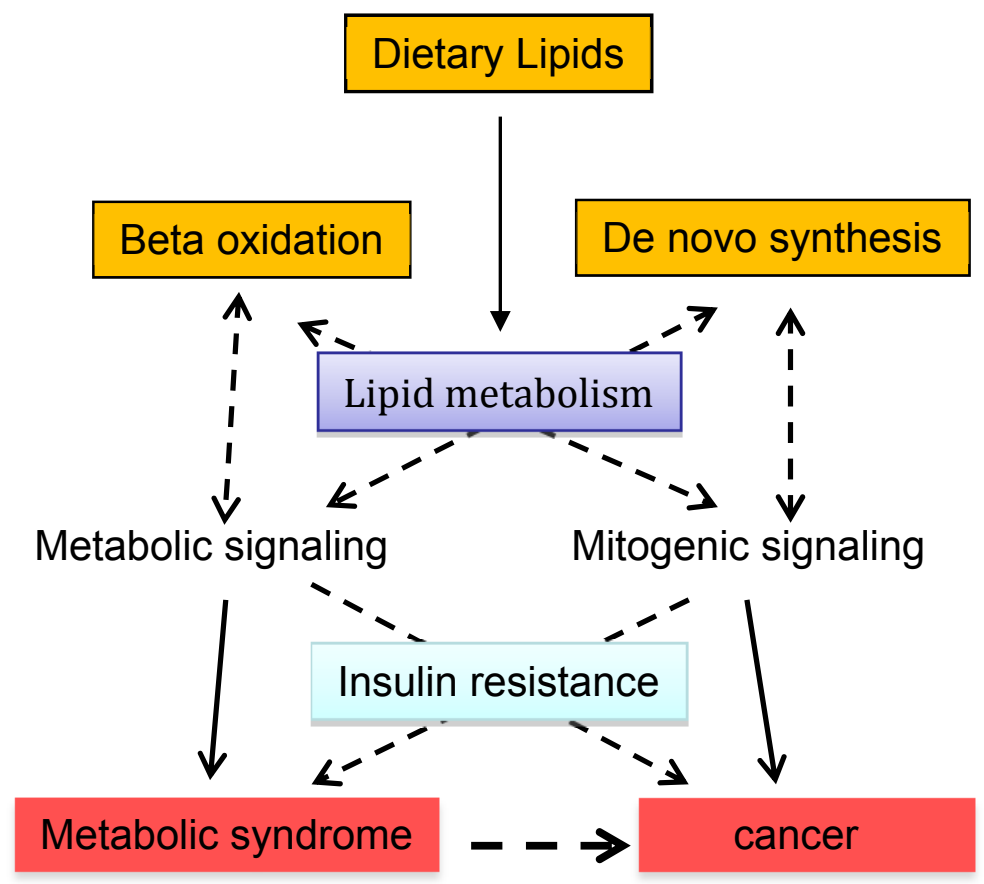

Figure 1. Inter-relationship between lipid metabolism, metabolic syndrome and cancer

\section{Dietary lipids and metabolic diseases}

\subsection{Dietary lipids and metabolic syndrome}

MS, also known as syndrome $X$, or the insulin resistance syndrome, is a combination of medical disorders comprising an array of metabolic risk factors including central obesity, dyslipidemia, hypertension, glucose intolerance, and insulin resistance[7]. The worldwide prevalence of MS causes lots of health problems not only in the developed countries, but also in the developing countries as well. Individuals with MS are at high risk for diabetes and cardiovascular disease. Increasingly accumulated evidence shows that aberrations in lipid metabolism are the central to the etiology of MS. 
As one of the most abundant lipid species and major components of very-low-density lipoprotein (VLDL) and chylomicrons, triacylglycerols (TAG) play an important role in metabolism as energy sources. It can be acquired from de novo synthesis in liver or dietary lipids. Depending on the oil source, TAGs are either the main constituents of vegetable oils (typically more unsaturated) or animal fats (typically more saturated). Animal fat comprises about $40 \%$ of the energy intake in the human diet in Western countries, and a high proportion of this is TAG. Fat tissue, liver and intestine are the major places where TAG is synthesized and stored. There is also some intracellular storage of TAG e.g. in the muscle and brain cells. The storage of TAG can be replenished from dietary fat, or by endogenous synthesis of fat from carbohydrates or proteins, which mainly takes place in the liver.

The overabundance of nutrients such as lipids in obesity and caloric surplus leads to aberrant lipid management and ectopic fat accumulation (i.e., "lipotoxicity"), which is a fundamental component of metabolic disease and insulin resistance $[8,9]$.

\subsubsection{Fatty acids and insulin resistance}

Insulin resistance is the center underlying the different metabolic abnormalities in the metabolic syndrome, in which pathophysiological conditions insulin becomes less effective in lowering blood glucose. Insulin resistance can be induced by various environmental factors, including dietary habits. Muscle, liver and fat are the three major tissues for maintaining blood glucose levels. In the presence of insulin, fat and muscle cells absorb glucose, and the liver regulates glucose levels by reducing its secretion and increasing its storage in the form of glycogen. However, in the condition of insulin resistance, glucose uptake by muscle and fat cells is disrupted, and glycogen synthesis and storage are also reduced in liver cells, resulting in failure of suppressing glucose production and releasing into the blood. Impaired glucose metabolism is associated with molecular alterations of insulin signaling, which is particularly well characterized in muscle [10].

Insulin also facilitates the uptake and storage of amino acids and fatty acids by converting them to protein and lipid, respectively. Besides the diminished glucose- lowering effects, insulin resistance also causes reduced actions of insulin on lipids and results in decreased uptake of circulating lipids and increased hydrolysis of stored triglycerides and, as a consequence, elevates free fatty acids in the blood plasma. Elevated levels of free fatty acids and triglycerides in the blood and tissues have been reported to contribute to impaired insulin sensitivity in many studies [11, 12]. Increased contents of fatty acids and their metabolites cause phosphorylation of insulin receptor substrate 1 (IRS-1) at serine, which blocks IRS-1 tyrosine phosphorylation and the associated activation of phosphatidylinositol3' kinase (PI3K) activity, and results in a decreased translocation of the glucose transporter GLUT4 to membrane of muscle and liver cells [13, 14].

The conversion of fatty acids to acetyl-CoA, the process known as $\beta$-oxidation, mainly occurs in the mitochondria. Defects in mitochondrial fatty acid oxidation and in adipocyte fat metabolism may increase fatty acid content in muscle and liver, which, in turn, cause impaired transport of glucose and defective glycogen synthesis in muscle, and sustained 
output of glucose from the liver, which finally lead to hyperinsulinemia and insulin resistance. In addition, oxidative stress and cytokine induction in liver may lead to the development of nonalcoholic fatty liver (NAFLD). Considerable experimental evidence suggests that increased hepatic fat synthesis contributes to nonalcoholic steatohepatitis and associated insulin resistance [15].

Dietary fat composition is the major sources of free fatty acids in the blood and tissue. Consumption of high fat diets is strongly and positively associated with overweight that, in turn, deteriorates insulin sensitivity, particularly when the excess of body fat is located in abdominal region. Epidemiological evidence and experimental animal studies clearly show that saturated fat significantly worsen insulin resistance, while monounsaturated (MUFA) and polyunsaturated fatty acids (PUFA) improve it through modifications in the composition of cell membranes, and an elevated ratio of saturated fats to unsaturated fats is a risk factor for MS [16, 17].

A multicenter study has shown that shifting from a diet rich in saturated fatty acids (SFAs) to one rich in monounsaturated fat improves insulin sensitivity in healthy people [18]. Substitution of unsaturated fat for saturated fat reduces both LDL cholesterol and plasma triglycerides in insulin resistant individuals [16]. Several early cross- sectional studies have found a positive association between saturated fat intake and hyperinsulinaemia, and insulin resistance $[19,20]$, while polyunsaturated fat intake was inversely associated with plasma insulin levels whereas linoleic acid intake was positively associated with fasting plasma insulin concentrations, and increased unsaturated fat intake is associated with improved insulin sensitivity [16, 21].

In addition, numerous observations in rodent and cell culture models as well as obese and diabetic humans have shown that chronic lipid exposure is associated with insulin resistance [22-24], and fatty acid composition in body tissues is related to the incidence of diabetes [25]. Particular, intramuscular or hepatic content of TAG, diacylglycerol (DAG), or ceramide is negatively correlated with insulin sensitivity [26-28], which may be caused by disrupted insulin-stimulated translocation of the GLUT4 by ectopic accumulation of TAG and other lipid molecules in liver and muscle.

More and more evidence suggests that ceramide, composition of sphingosine and fatty acid and found in high concentrations within the cell membrane, plays a critical role in insulin resistance [29]. Both in vitro and in vivo studies have produced a large body of data implicating that accumulation of ceramide and its metabolites is associated with nutrientinduced pathogenesis of insulin resistance and metabolic diseases, including diabetes, cardiomyopathy, and atherosclerosis [28-30]. In cultured cells, ceramide inhibits insulinstimulated glucose uptake by blocking translocation of the glucose transporter GLUT4, and glycogen synthesis [31, 32]. These effects are due to the ability of ceramide to block activation of $\mathrm{Akt} / \mathrm{PKB}$, a serine/threonine kinase that is activated by insulin and growthfactors. It is also found that accumulation of ceramide may impair mitochondrial function by altering mitochondrial membrane permeability, inhibiting electron transport chain intermediates, and promoting oxidative stress [33]. 
In rodents, inhibition of ceramide de novo synthesis pathway by serine palmitoyltransferase inhibitor myriocin improves insulin sensitivity and prevents insulin resistance associated metabolic diseases [30, 34-36]. In humans, it is well documented the association of ceramides accumulation in peripheral tissues, including muscle and fat, of obese subjects with insulin resistance [37-40].

\subsubsection{Fatty acids and cardiovascular diseases}

Since the 1950s, it has long been believed that consumption of foods containing high amounts of SFAs, including meat fats, milk fat, butter, lard, coconut oil, etc, is not only a risk factor for dyslipidemia and insulin resistance, but also a risk factor for cardiovascular diseases. However, recent evidence from systematic reviews, meta-analyses and prospective cohort studies indicates that SFAs alone maybe not associated with an increased risk of cardiovascular disease. A randomized controlled dietary intervention trial that compared a carbohydrate restricted diet to a low fat diet over a 12-week period in overweight subjects with atherogenic dyslipidemia found that carbohydrate restriction, rather than a low fat diet may improve features of MS and cardiovascular risk [41]. In a recent cross-sectional study conducted in Japanese to exam the relationship between dietary ratio of PUFA to SFA with cardiovascular risk factors and MS, the data showed that dietary polyunsaturated to saturated fatty acid ratio was significantly and inversely related to serum total and LDL cholesterol, but did not significantly relate to single metabolic risk factors or the prevalence of MS [42].

However, on the other hand, some SFAs such as stearic acid and fatty acids found in milk and milk products appear to be beneficial and may diminish the risk for cardiovascular disease. In a systematic review, after comparing with those of trans, other saturated, and unsaturated fatty acids (USFAs), Hunter et al [43] found that stearic acid raised LDL cholesterol, and compared with USFA, stearic acid lowered HDL cholesterol and increased the total cholesterol/HDL cholesterol ratio [43].

Palmitoleic acid (cis-16:1, n-7) has been linked to both beneficial metabolic effects. It has been reported that adipose-produced cis-palmitoleate directly improved hepatic and skeletal muscle insulin resistance and related metabolic abnormalities, and suppressed hepatic fat synthesis as well [44]. A prospective cohort study showed that circulating transpalmitoleate (trans-16:1, n-7) is associated with lower insulin resistance, decreased presence of atherogenic dyslipidemia, and incidence of diabetes incidence [45] suggesting metabolic benefits of dairy consumption. There is also strong evidence collected by systematic review and meta-analysis of randomized controlled trials showing that consumption of polyunsaturated fat as a replacement for saturated fat alleviates coronary heart disease risk [46]. While many studies have found that replacement of saturated fats with polyunsaturated fats in the diet produces more beneficial outcomes on cardiovascular health $[47,48]$, the effects of substituting monounsaturated fats or carbohydrates are still unclear.

\subsubsection{Omega-3 PUFAs and metabolic syndrome}

Omega-3 PUFAs (also called $\omega-3$ fatty acids or $n-3$ fatty acids are commonly found in marine and plant oils, which contain a double bond at the third carbon atom from the 
methyl-end of the carbon chain. These PUFAs, including $\alpha$-linolenic acid (ALA, 18:3, n-3), eicosapentaenoic acid (EPA, 20:5, n-3) and docosahexaenoic acid (DHA, 22:6, n-3), are considered as essential fatty acids because they can not be de novo synthesized by the human body. In human diets, ALA is usually derived from botanical sources such as perilla, flaxseed, canola, rapeseed, soybean, linseed and walnut. EPA and DHA are found in fish and some other sea foods [49]. Recent researches have shown that, while diets rich in saturated fatty acids (SFAs) are associated with an increased prevalence of obesity and type 2 diabetes, supplement of omega-3 PUFAs rich in eicosapentaenoic acid (EPA) and docosahexaenoic acid (DHA) has anti-inflammatory and anti-obesity effects and protect against metabolic abnormalities [50].

Earlier epidemiologic observations showed the beneficial properties of n-3 PUFAs in populations consuming large amounts of fatty fish and marine mammal oils [51]. Later studies showed that a 3-wk supplement with fish oil rich in n-3 PUFA in healthy humans resulted in improved sensitivity to insulin, higher fat oxidation, and increased glycogen storage [52]. Most subsequent studies confirmed these effects and observed that supplementation with n-3 PUFAs, either EPA or DHA alone, or with their combination in fish oil, has favorably effects on many adverse serum and tissue lipid alterations related to the metabolic syndrome by reducing levels of fasting and postprandial serum triacylglycerols and free fatty acids $[53,54]$. Some of the effects of n-3 PUFAs on lipid and lipoprotein metabolism could remain in subjects who become overtly diabetic.

In addition, other recognized benefits of n-3 PUFAs include a reduction in inflammatory status, decreased platelet activation, mild reduction in blood pressure, improved endothelial function, and increased cellular antioxidant defense, all of which may prove particularly favorable in overweight, hypertensive patients [55]. Furthermore, supplementation with fish oil also blunted the sympathetic activity elicited by mental stress in healthy volunteers [56]. However, the beneficiary effects of n-3 PUFA supplementation on cardiovascular risk prevention are association with other components of lifestyle, ie, weight control, regular physical activity, and consumption of other dietary ingredients contributing to risk reduction [57].

The mechanisms underlying beneficiary effects of use n-3 PUFAs/fish oils or a combination of EPA and DHA have been extensively analyzed. Studies in animal and humans have demonstrated that, in addition to be used as fuels and structural components of the cell, the dietary intake of marine fish oil is also effective in lowering both triglyceride (Tg) and VLDL-Tg concentration in experimental animals and normal and hyper- triglyceridemic men [58, 59], which might be related to decreased mRNA encoding several proteins involved in hepatic lipogenesis including SREBP1, and enhanced fatty acid oxidation throughout a peroxisome proliferator- activated receptors (PPARs) -stimulated process [60, 61]. Moreover, n-3 PUFAs elevate the fatty acid composition of membrane phospholipids that modify membrane-mediated processes such as insulin transduction signals, activities of lipases and biosynthesis of eicosanoids [62].

Furthermore, dietary fish oil consumption normalizes the function of many tissues or cells involved in insulin sensitivity in the sucrose-rich diet (SRD) fed rats. It reverses dyslipidemia and improves insulin action and adiposity by reducing adipocytes cell size, 
increasing insulin sensitive and decreasing the release of fatty acids. Both oxidative and non-oxidative glucose pathways are improved in muscle. In isolated beta cells, lipid contents and glucose oxidation return to normal [63]. All these effects lead to the improvement of glucose- stimulated insulin secretion and muscle insulin insensitivity.

Adipose tissue plays a key role in the development of MetS and improvement of adipose tissue function is specifically linked to the beneficial effects of n-3 PUFA [64]. In accordance with the general anti-inflammatory action, n-3 PUFA supplementation induces production and secretion of adiponectin [65], the major adipokine exerting an insulin-sensitizing effect, and prevents adipose tissue hyperplasia and hypertrophy, and induces mitochondrial biogenesis in adipocytes [66], which effects maybe mediated by n-3 PUFA induced AMP-activated protein kinase (AMPK), a metabolic sensor controlling intracellular metabolic fluxes [64].

\subsection{Dietary lipids and cancer}

Case-control and cohort studies have found positive associations between several cancers such as prostate cancer[67], ovarian cancer[68], breast cancer[69], colon cancer[70] etc, and an intake of foods with high levels of saturated fats, such as red meat, eggs, and dairy products. However, controversial results have also been reported about the role of high fat diet in carcinogenicity [71,72]. This is largely due to the complexity of the diet, not only the fat components such as SFA, MUFA, and PUFA may vary among people in different regions, but also other non-fat nutrients may also alter the function of fat. Therefore only preclinical animal studies with clearly-defined fat composition may help elucidate the causal relationship between dietary fat and cancer. Up to now, it is generally accepted that cis-MUFA and omega-3 PUFAs are inversely associated with the increased risk of cancer, while SFA and omega-6 PUFAs are associated with the development of cancer [73]. However, physiologically, the metabolism of fatty acids are connected, any results based on a single fatty acids may be incomprehensive, therefore a fat containing diet with elevated MUFA and low ratio of omega-6/omega-3 fatty acid is suggested to be associated with cancer prevention and protection [74]. Interested readers are advised to read recent review articles about the association of dietary lipids with prostate [75] and breast cancer [76], and potential mechanisms for the association of dietary lipids with cancer [77-79].

\subsubsection{Saturated fatty acids}

Recent studies have shown that high fat diet with saturated animal fat as major fat in the diet is associated with several cancer such as prostate cancer [67], colon cancer [80], ovarian cancer [68] and breast cancer [81] etc, whereas high fat diet with plant oils is not associated with cancer risk, however this may not be true, plant oils high in omega- 6 fatty acids may be risk factors for cancer, which will be discussed in the polyunsaturated fatty acid section.

\subsubsection{Monounsaturated fatty acids}

It has been found that cancer incidence in the Mediterranean countries, where the main source of fat is olive oil, is lower than in other areas of the world. Such effects may be due to 
the main MUFA in olive oil, oleic acid, and to certain minor compounds such as squalene and phenolic compounds [82]. Recent studies have also shown that canola oil, with high MUFA, oleic acid, can decrease colon and breast cancer incidence significantly [83, 84]. Although the authors suggested that such effect may be caused by omega- 3 fatty acids, ALA, as high as $10 \%$ in canola oil, however, the role of oleic acid, as high as $61 \%$ in canola oil, cannot be excluded. So far, no epidemiological studies or animal studies can clearly demonstrate the preventive effect of MUFA on cancer, However, in vivo analysis of the fatty acid composition of the adipose tissue of breast cancer and healthy women showed that elevated adipose MUFA, oleic acid, are associated with reduced odds of breast cancer [85]. Although the mechanism underling the protective function of oleic acid on cancer is, so far, not clear, it has been found that oleic acid, when complexed with the molten globule form of alpha-lactalbumin ( $\alpha$-LA), acquires tumoricidal activity [86]. Carrillo et al found that oleic acid can inhibit store-operated $\mathrm{Ca}(2+)$ entry (SOCE), a $\mathrm{Ca}(2+)$ influx pathway, involved in the control of multiple cellular and physiological processes including cell proliferation, thus regulating the growth of colon carcinoma cells [87].

\subsubsection{Polyunsaturated fatty acids}

Increasing evidences from animal and in vitro studies indicate that populations who ingest high amounts of omega-3 fatty acids in their diets have lower incidences of breast, colon, and, perhaps, prostate cancers. Paola et al. [88] used MTT viability test and expression of apoptotic markers to evaluate the effect of PUFAs on cancer growth, and their results indicated that EPA and DHA might induce modifications of tumor cell membrane structure leading to an obviously decreased induction rate of breast cancer. Menéndez et al. [89] also reported that omega-3 PUFAs ALA suppresses the overexpression of HER2, which plays an important role in aetiology, progression and chemosensitivity of various types of human cancers, suggesting that ALA is a potential anticancer agent. However, the clinical roles of omega-3 PUFAs may rely not only on the absolute content but also on the proportion of omega-3 PUFAs to omega-6 PUFAs in the cells due to the inverse biological functions of these two series of PUFAs. A higher omega-6/omega-3 PUFAs ratio contributes to many diseases including cancer, cardiovascular and inflammation. Reducing the omega-6/omega3 PUFAs ratio can help lower the risk of initiation and development of cancer. Berquin et al. established a prostate-specific phosphatase and tension homolog (PTEN) knockout mouse model, and the result demonstrated that a dietary ratio of omega-6/omega-3 PUFA lower than 5 was effective in suppressing tumor growth, and extending animal lifespan [90] The recent research suggested that a balanced ratio of omega-6/omega-3 PUFAs (1:1) exerts a beneficial effects on cell function and physiology [91].

\subsubsection{Potential mechanisms of the association of dietary lipids with cancer}

Although it has been generally accepted that dietary lipids are associated with carcinogenesis and the development of cancer, the detailed mechanism is still far from clear. When lipids are digested and absorbed by small intestine mucosa cells, they can be transported to adipocytes for storage, or used for energy production by peripheral cells 
through fatty acid $\beta$-oxidation. They can also be used for membrane lipid biosynthesis. Upon environmental stimulus, these lipids may be hydrolyzed and free fatty acids are released. Omega-6 PUFAs such as ARA released from membrane lipids will be converted to normal eicosanoids, and regulate cellular physiology; however elevated levels of these eicosanoids may accelerate cell proliferation and lead to inflammation and carcinogenesis, etc [92]. Whereas omega-3 PUFAs such as EPA, when released from membrane lipids, may be converted to eicosanoids with opposite activity to the product of omega- 6 fatty acids, which inhibit cell proliferation and COX-2 activity, thus providing cancer preventive function [93]. Another mechanism of regulation of cancer initiation and development may be elucidated by fatty acid signaling pathway through its receptors. In particular, two transcription factors, sterol regulatory element binding protein-1c (SREBP-1c) and peroxisome proliferator activated receptor alpha (PPAR alpha), have emerged as key mediators of gene regulation by FA $[94,95]$. SREBP-1c induces a set of lipogenic enzymes in liver. PUFA, but not SFA or MUFA, suppressES the induction of lipogenic genes by inhibiting the expression and processing of SREBP-1c. Thus inhibits the de novo lipogenesis of fatty acids, which is of particular importance for cancer cells [96].

PPAR alpha plays an essential role in metabolic adaptation to fasting by inducing the genes for mitochondrial and peroxisomal FA oxidation as well as those for ketogenesis in mitochondria. FAs released from adipose tissue during fasting are considered as ligands of PPAR alpha. Dietary PUFA, except for 18:2 n-6, are likely to induce FA oxidation enzymes via PPAR alpha as a "feed-forward " mechanism. PPAR alpha is also required for regulating the synthesis of highly unsaturated FA, indicating pleiotropic functions of PPAR alpha in the regulation of lipid metabolic pathways. Thus, in addition to its inhibition of fatty acid biosynthesis through SREBP, omega-3 fatty acids induce fatty acid degradation through PPAR alpha, in so doing, they regulate fatty acid metabolism and metabolic diseases. Multiple mechanisms of omega-3 fatty acids mediated inhibition of cancer may include suppression of neoplastic transformation and cell growth, and enhanced apoptosis and antiangiogenicity etc [97].

\section{De novo lipogenesis in metabolic disease}

\subsection{De novo lipogenesis in metabolic syndrome}

De novo fatty acid biosynthesis occurs in essentially all cells, but adipose tissue and liver are the major sites. The first committed step in fatty acid synthesis is catalyzed by fatty acid synthase (FAS), a multifunctional cytosolic protein that primarily synthesizes palmitate. Variations in FAS expression and enzyme activity have been implicated in insulin resistance and obesity in humans [98]. A circulating form of FAS has been reported as a biomarker of metabolic stress and insulin sensitivity. In humans it changes with weight loss and may reflect improved insulin sensitivity [99].

Fatty acid elongation is catalyzed by Elovl (elongation of very long-chain fatty acid) proteins. Elovl6 is thought to be involved in de novo lipogenesis and is regulated by dietary, 
hormonal and developmental factors. Mice with Elov6 deficiency are obese but protected from insulin resistance [100, 101].

Citrate produced by the tricarboxylic acid cycle in mitochondria is converted by ATP-citrate lyase (ACL) to acetyl-CoA, which is next converted to malonyl-CoA by acetyl CoA carboxylase (ACC). Malonyl-CoA is a potent inhibitor of carnitine- palmitoyl transferase 1 (CPT1), which transports FAs into the mitochondria for oxidation, thus plays a key role in the regulation of both mitochondrial fatty acid oxidation and fat synthesis. ACC catalyzes a key rate-controlling step in both de novo lipogenesis and fatty acid oxidation. The absence of ACC decreases the cellular concentration of malonyl-CoA, removes the inhibition of CPT1 and maintains FA oxidation. In rats with NAFLD, suppression or knockdown of ACC isoforms significantly reduced hepatic malonyl-CoA levels, lowered hepatic lipids including long-chain acyl-CoAs, DAG, and triglycerides, and improved hepatic insulin sensitivity [102].

Lipogenesis and FA oxidation are highly integrated processes. Studies in genetically modified mice have demonstrated that inhibition of FA synthesis and storage is associated with upregulation of FA oxidation [103]. For examples, knockout the diacylglycerol acyltransferase (DGAT), an enzyme that catalyses the final acylation step of TAG synthesis, reduced fat deposition and protected mice against diet- induced obesity and, in the meanwhile, elevated mice energy expenditure and increased activity, suggesting a correlation of disrupted FA storage and increased FA oxidation [104, 105]. Similarly, deletion of acetyl-CoA carboxylase 2 (ACC2), an isoform of ACC and key enzyme for de novo FA synthesis, leads to a lean mouse with increased FA oxidation [106].

As a major component of the metabolic syndrome, NAFLD characterizes with the accumulation of TAGs in hepatocytes, and development of steatohepatitis, cirrhosis, and hepatocellular carcinoma. FAs stored in adipose tissue and newly made through liver de novo lipogenesis are the major sources of TAGs in the liver [107].

Lipogenesis is also an insulin- and glucose-dependent process that is under the control of specific transcription factors. SREBP1 is such a transcription factor and activates most genes involved in FA synthesis. It occurs in two isoforms, SREBP1a and 1c, through alternative splicing. SREBP-1c is highly expressed in the WAT, liver, adrenal gland, brain, and muscle and regulates the expression of many of the genes involved in de novo FA and TAG synthesis including ACC and FAS [108, 109]. Insulin increases lipogenesis through activating SREBP-1c that is dependent on the mammalian target of rapamycin (mTOR) complex 1 (mTORC1) [110]. SREBP1 gene expression is decreased in adipose tissue of obese subjects and the aberrant activation of SREBPs may contribute to obesity-related pathophysiology in various organs, including cardiac arrhythmogenesis and hepatic insulin resistance.

Lipogenesis is also regulated by glucose activated carbohydrate response element-binding protein (ChREBP), which induces gene expression of liver-type pyruvate kinase, a key regulatory enzyme in glycolysis; this enzyme in turn provides the precursors for lipogenesis [111]. ChREBP also stimulates expression of genes involved in lipogenesis [112] including SREBP-1c, which in turn activates glycolytic gene expression, promoting glucose 
metabolism, and lipogenic genes in conjunction with ChREBP [113]. ChREBP knockout mice show decreased liver triglyceride but increased liver glycogen content indicating that ChREBP may regulate metabolic gene expression to convert excess carbohydrate into triglyceride rather than glycogen [114]. In addition, complete inhibition of ChREBP in ob/ob mice reduces the effects of the MS such as obesity, fatty liver, and glucose intolerance, indicating it as a potential target for treatment of MS [115].

\subsection{De novo lipogenesis in cancer}

Enhanced flux of glucose derivatives through glycolysis, which sustain the redirection of mitochondrial ATP to glucose phosphorylation, and de novo FA synthesis is a hallmark of aggressive cancers. Although most normal cells use FA from dietary lipids, tumor cells de novo synthesize more than $95 \%$ of lipids required for cell proliferation despite having enough nutritional supply of lipids. Lipogenic enzymes such as, FAS, ACC, and ACL involved in FA biosynthesis, glycerol-3-phosphate dehydrogenase involved in lipid biosynthesiss, and SREBP1, the master regulator of lipogenic gene expression, are found to be overexpressed in a number of cancer or cancer cells, such as prostate cancer [116], ovarian cancer [117], breast cancer [118], lung cancer [119], colon cancer [120], and etc. Some research has been carried out to provide insights into the molecular mechanism of the association of lipogenesis and cancer. In this chapter we focused on three main lipogenic genes: FAS, ACC, and ACL.

\subsubsection{Fatty acid synthase}

High levels of FAS expression have been found in many human cancers, including prostate cancer [121], ovarian cancer [117, 122], breast cancer [123], bladder cancer [124], colon cancer [125] mantle cell lymphoma [126], and etc. However, the cellular mechanism by which FAS is up-regulated in cancer cells is not fully understood. Nevertheless a few studies suggested that steroid hormones and human epidermal growth factor receptor (HER) family ligands, especially HER2 could increase FAS expression via the PI3K/Akt or mitogen-activated protein kinases (MAPK) pathways[117]. Recently Mukherjee et al [117] demonstrated that bioactive lipid lysophosphatidic acid (LPA) induces FAS expression and lipogenesis through LPA2-G12/13-Rho-SREBP signaling pathway in ovarian cancer cells. Moreover over-expression of FAS in non-cancerous epithelial cells is sufficient to induce a cancer-like phenotype through the induction of HER1/HER2 [127], therefore FAS over-expression may play a role in carcinogenesis. Furthermore FAS overexpression is found to be associated with the advanced stage of colorectal cancer and liver metastasis, thus it may also play a role in the progression of cancer [128]. So far abundant evidences have shown that FAS contributes to both tumorigenesis and metastasis, and it becomes an ideal target for cancer therapy. In deed inhibition of FAS activity by FAS specific inhibitors or siRNA can significantly inhibit cancer or cancer cell growth, induce cancer cell apoptosis, and reduce the metastasis of several cancers[124, 129]. Both synthetic chemicals and natural products of 
FAS inhibitors have been developed [123[130], and the recent progress in developing FAS inhibitors as cancer drugs has been reviewed by Pandey et al [131].

\subsubsection{ATP-Citrate lyase and acetyl CoA carboxylase}

Apart from FAS, other key lipogenic enzymes for de novo FA biosynthesis include ACL and ACC. While ACL produce the substrate acetyl-CoA from glycolytic product citrate, ACC activates the substrate to generate malanyl-CoA, the building block for fatty acid synthesis. Both ACC and ACL have been found to be over-expressed in many cancers such as breast, liver, lung, ovarian, prostate and leukemia cancers [132, 133]. Inhibition of either ACL or ACC induces growth arrest and apoptosis in several cancer cell lines [134-136]. The potential mechanism of ACL overexpression in tumorigenesis is through PI3K/AKT and MAPK signaling pathway [135, 137]. Yoon et al [138] found that the major mechanism of HER2mediated induction of ACC alpha in breast cancer cells is translational regulated primarily through mTOR signaling pathway. While Mukherjee et al [117] found that LPA induced induction of ACC in ovarian cancer cells is through LPA2-Gq-PLC-AMPK signaling pathway. Many small molecule inhibitors for ACL and ACC have been developed as potential therapeutic agents for cancer [133, 139].

\section{Phospholipids metabolism in metabolic diseases}

Phospholipids are polar lipids as major component of membrane structure and some intracellular complex such as lipoproteins. Enzymes involved in the metabolism of phospholipids include phospholipase $\mathrm{A}_{2}\left(\mathrm{PLA}_{2}\right)$, phospholipase C (PLC), phospholipase D (PLD), and lysophospholipase D (autotoxin), and alterations of these enzymes have been found to be linked with metabolic diseases, such as MS and cancer. In addition, the intermediates or end products of phospholipid metabolism such as phosphatidic acid (PA), DAG, LPA, sphingosine-1-phoshate (S-1-P), and free fatty acid arichidonic acid (ARA), are also involved in the pathogenesis of metabolic diseases.

\subsection{Phospholipid metabolism in metabolic syndrome}

Phosphatidylcholine (PC) is the most abundant phospholipids in animal cells. Blocking Sadenosylmethionine (SAMe) or PC synthesis in C. elegans, mouse liver, and human cells have been found to cause elevated SREBP-1-dependent transcription and lipid droplet accumulation [4], suggesting nutritional or genetic conditions limiting SAMe or PC production may activate SREBP-1, and contribute to human metabolic disorders.

Phosphatidylethanolamine (PE) is another abundant phospholipid in mammals. PE and its downstream signaling events play an important role in the heart function, and alteration in the asymmetrical transbilayer distribution of PE in sarcolemmal membranes during ischemia causes sarcolemmal disruption [140]. Moreover, abnormalities in the molecular species profile of PE may contribute to membrane dysfunction and defective contractility of the diabetic heart [141, 142]. 
SREBPs may play critical roles in phospholipid homeostasis and lipotoxic cardiomyopathy. Dysregulated phospholipid signaling that alters SREBP activity has been reported to contribute to the progression of impaired heart function in flies and also act as a potential link to lipotoxic cardiac diseases in humans [143]. Thus the role of SREBPs in modulating heart function and its associated phospholipid signaling maybe a candidate target for future therapies for obesity- and diabetes- related cardiac dysfunction.

\subsection{Phospholipid metabolism in cancer}

An aberrant choline phospholipid metabolism is another major hallmark of cancer cells. In deed alterations of choline phospholipid metabolism have been reported in ovarian cancer and also in breast cancer [144, 145]. Altered choline phospholipid metabolism in ovarian cancer has been found to be linked with the regulation of FAS. Because the drop in the level of PC (59\%) was significantly correlated with a drop in de novo synthesized FA levels, PC was identified as a potential noninvasive magnetic resonance spectroscopy-detectable biomarker of FAS inhibition in vivo [146]. Phospholipids and their metabolism have been found to be involved in ovarian cancer in several forms, including LPA, PLA2, PLD, and autotoxin (ATX). Although aberrant phospholipid metabolism has been found in other cancers, the most detailed research work has been carried out using ovarian cancer as a model, so in this section we summarized the recent advances in the research of phospholipid metabolism and ovarian cancer.

\subsubsection{Lysophosphatidic acid}

The LPAs, with their various FA side chains, are the constituents of a growth-stimulating factor-ovarian cancer activating factor-that has been identified from ascites in patients with ovarian cancer [147]. As a bioactive compound, LPA works to induce cell proliferation or differentiation, prevents apoptosis induced by environmental stress or stimuli, induce platelet aggregation and smooth muscle contraction, and stimulate morphological changes, adhesion and migration of cells. It thus is involved in a broad range of biologic processes in a variety of cellular systems [148, 149]. As an established mitogen, LPA also promotes the invasiveness of hepatoma cells into monolayers of mesothelial cells, and stimulates proliferation of ovarian and breast cancer cell lines even in the absence of other growth promoters such as serum. Furthermore, LPA stimulates rapid neurite retraction and rounding of the cell body in serum-deprived neuroblastoma cells [150], and plays a critical role in regulation of gene expression in normal and neoplastic cells. It is a potent modulator of the expression of genes involved in inflammation, angiogenesis, and carcinogenesis such as interleukin [151-154], vascular endothelial growth factor (VEGF) [155], urokinase plasminogen activator [156], and cyclooxygenase-2 [157]. Thus LPA may contribute to cancer progression by triggering expression of those target genes, resulting in a more invasive and metastatic microenvironment for tumor cells [152, 158]. A significant increase in the expression of LPA receptors (LPA2 and LPA3) with VEGF was found by Fujita et al. [159], who suggested that LPA receptors might be involved in VEGF expression mediated 
by LPA signals in human ovarian oncogenesis. The recent identification of metabolizing enzymes that mediate the degradation and production of LPA and the development of receptor selective-analogs has opened a potential new approach to the treatment of ovarian cancer [160]. LPA also stimulates VEGF expression independent of hypoxia-inducible factor 1 (H1F1) and promotes tumor angiogenesis by activation of c-Myc and Sp-1 transcription factors [161]. A very recent study shows that LPA induces de novo lipogenesis through LPA2-G12/13-SREBP-FAS, and LPA2-G(q)-AMPK-ACC signaling pathway.

\subsubsection{Phospholipase $A_{2}$}

The PLA 2 enzyme has been implicated in the activation of cell migration and the production of LPA in ovarian carcinoma cells [162]. Autonomous replication and growth-factorstimulated proliferation of ovarian cancer cells are highly sensitive to inhibition of calciumindependent PLA2 (iPLA2), but are refractory to inhibition of cytosolic PLA2 [162]. Activation of iPLA2 plays a critical role in cell migration, which is involved in many important biologic processes such as development, the immunologic and inflammatory responses, and tumor biology [162]. When ovarian cancer cells were grown under growthfactor-independent conditions, suppression of iPLA2 activity led to an accumulation of cell populations in both the $S$ and the G2/M-phases [163]. Supplementation with exogenous growth factors such as LPA and epidermal growth factor in culture released the S-phase arrest, but did not affect the G2/M arrest associated with inhibition of iPLA2. In addition to the prominent effect on the cell cycle, inhibition of iPLA 2 also induced weak-to-modest increases in apoptosis [163]. Downregulation of iPLA 2 owith lentivirus-mediated RNA interference targeting iPLA 2 oexpression inhibited cell proliferation in culture and decreased tumorigenicity of ovarian cancer cell lines in athymic nude mice [163]. Recently iPLA2 has been found to play a role in breast cancer metastasis as iPLA 2 deficiency protects breast cancer from metastasis to the lung [164].

\subsubsection{Phospholipase D}

PLD, a family of signaling enzymes that most commonly responsible to generate most lipid second messenger phosphatidic acid (PA), is found in diverse organisms from bacteria to humans and functions in multiple cellular pathways. It has been increasingly recognized as a critical regulator of cell proliferation and tumorigenesis and the expression and activity of PLD are elevated in many different types of human cancers.

In ovarian cancer cells, PLD is involved in the formation of PA, which may be further converted to LPA by PLA2. It was suggested that PLD is also involved in cancer progression and metastasis and elevated PLD expression has been reported in various cancer tissues [165]. Moreover, PLD was found to stimulate cell protrusions in v-Src-transformed cells [166]. Furthermore, PLD activity was elevated by the integrin receptor signaling pathway in OVCAR-3 cells, and PLD blocking was found to inhibit integrin-mediated Rac translocation in, and the spreading and migration of, OVCAR-3 cells [167]. Thus, the PLD-PA-Rac pathway plays an important role in the metastasis of cancer cells, and might provide a 
connection for integrin and PLD-mediated cancer metastasis [167]. A new mechanism has also been suggested for PLD and PA mediated carcinogenesis through $W n t / \beta$-catenin signaling network [168].

\subsubsection{Autotaxin}

The ATX protein is a member of the ectonucleotide pyrophosphatase and phosphodiesterase family of enzymes, but unlike other members of this group, ATX possesses lysophospholipase D activity. This enzyme hydrolyzes lysophosphatidylcholine (LPC) to generate bioactive lipid LPA, which is an important signaling molecule regulates a variety of biological process through its receptors. ATX is essential for normal development and is implicated in various physiological processes. It also acts as a potent tumor growth factor and mitogen that is, associated with pathological conditions such as cancer, pain and fibrosis. Exogenous addition of VEGF-A to cultured cells induces ATX expression and secretion, resulting in increased extracellular LPA production [169]. This elevated LPA, acting through LPA4, modulates VEGF responsiveness by inducing VEGF receptor 2 expression. Downregulation of ATX secretion in SKOV3 cells significantly attenuates cell motility responses to VEGF, ATX, LPA, LPC [169]. Through their respective G proteincoupled receptors, LPC and LPA have both been reported to stimulate migration [170]. LPC was unable to stimulate the cellular migration by itself, ATX had to be present. Knocking down ATX secretion, or inhibiting its catalytic activity, blocked cellular migration by preventing LPA production and the subsequent activation of LPA receptors [170].

\section{Summary}

As a combination of central obesity, dyslipidemia, and insulin resistance, MS is the central of world-wide prevalence of Type 2 Diabetes Mellitus (T2DM), cardiovascular diseases and inflammation. Current animal and clinical evidence strongly suggest that abnormal lipid metabolism is closely associated with onset of insulin resistance and cancer. Importantly, more and more evidence show that most of the components of the MS are linked in some way to the development of various cancers [171-173], although epidemiological studies linking the MS to cancer are highly required. Obesity and diabetes have been reported to be associated with breast, endometrial, colorectal, pancreatic, hepatic or renal cancer [174, 175]. The molecular links between MS and cancer are still unclear, but insulin/insulin-like growth factor (IGF) systems and associated intracellular signaling cascades may play an important role in mediating MS related cancers [173]. However, the mechanisms by which actually promote tumor cell growth in patients with MS need further investigation. Since lipids and their metabolites and metabolism pathways are related to metabolic diseases and cancer cell growth, we propose that lipids may link to MS and cancers and exploring the related molecules and understanding the underlying mechanisms will be helpful in developing potential therapies for both MS and cancer. Based on the discoveries of current research results, a diet with high amount of oleic acid and balanced ratio of omega-3/omega-6 PUFAs would be helpful for health and prevention of both MS and cancer. 


\section{Author details}

Fang $\mathrm{Hu}$

Center for Food Biotechnology, School of Food Science and Technology,

State Key Laboratory of Food Science and Technology, Jiangnan University, Jiangsu, China

Metabolic Syndrome Research Center, the Second Xiangya Hospital, Central South University, Changsha, China

Yingtong Zhang and Yuanda Song

Center for Food Biotechnology, School of Food Science and Technology,

State Key Laboratory of Food Science and Technology, Jiangnan University, Jiangsu, China

\section{Acknowledgement}

Fang $\mathrm{Hu}$ is supported by National Key Basic Research Program of China (973 Program) 2012CB524900, National Science Foundation of China (NSFC) grant 31071921, and NSFC grant 81170783 . Yuanda Song is supported by NSFC grant 81071685 , Open Project Program of State Key Laboratory of Food Science and Technology, Jiangnan University (SKLF-TS-201101), and starting grant from Jiangnan University.

\section{References}

[1] Kiebish M A, et al. (2008) Cardiolipin and electron transport chain abnormalities in mouse brain tumor mitochondria: lipidomic evidence supporting the Warburg theory of cancer. J Lipid Res. 49: 2545-56.

[2] Swinnen J V and G Verhoeven (1998) Androgens and the control of lipid metabolism in human prostate cancer cells. J Steroid Biochem Mol Biol. 65: 191-8.

[3] Lee C H, P Olson, and R M Evans (2003) Minireview: lipid metabolism, metabolic diseases, and peroxisome proliferator-activated receptors. Endocrinology. 144: 2201-7.

[4] Walker A K, et al. (2011) A conserved SREBP-1/phosphatidylcholine feedback circuit regulates lipogenesis in metazoans. Cell. 147: 840-52.

[5] Podo F, et al. (2011) MR evaluation of response to targeted treatment in cancer cells. NMR Biomed. 24: 648-72.

[6] Pegorier J P, et al. (1985) Effect of intragastric triglyceride administration on glucose homeostasis in newborn pigs. Am J Physiol. 249: E268-75.

[7] Eckel R H, S M Grundy, and P Z Zimmet (2005) The metabolic syndrome. Lancet. 365: 1415-28.

[8] Unger R H (2002) Lipotoxic diseases. Annu Rev Med. 53: 319-36.

[9] Unger R H and P E Scherer (2010) Gluttony, sloth and the metabolic syndrome: a roadmap to lipotoxicity. Trends Endocrinol Metab. 21: 345-52.

[10] Hotamisligil GS P P, Budavari A, Ellis R, White MF, Spiegelman BM. (1996) IRS-1mediated inhibition of insulin receptor tyrosine kinase activity in TNF-alpha- and obesity-induced insulin resistance. Science. 271(5249): 665-8. 
[11] Schinner S, et al. (2005) Molecular mechanisms of insulin resistance. Diabet Med. 22: 674-82.

[12] Storlien LH J A, Chisholm DP, Pascoe WS, Kraegen EW. (1991) Influence of dietary fat composition on development of insulin resistance in rats: relationship to muscle triglyceride and omega-3 fatty acids in muscle phospholipids. Diabetes. 40: 280-9.

[13] Shulman G I (2000) Cellular mechanisms of insulin resistance. J Clin Invest. 106: 171-6.

[14] Shulman G I (2004) Unraveling the cellular mechanism of insulin resistance in humans: new insights from magnetic resonance spectroscopy. Physiology (Bethesda). 19: 183-90.

[15] Postic C and J Girard (2008) Contribution of de novo fatty acid synthesis to hepatic steatosis and insulin resistance: lessons from genetically engineered mice. J Clin Invest. 118: 829-38.

[16] Riccardi G, R Giacco, and A A Rivellese (2004) Dietary fat, insulin sensitivity and the metabolic syndrome. Clin Nutr. 23: 447-56.

[17] Parillo M and G Riccardi (2004) Diet composition and the risk of type 2 diabetes: epidemiological and clinical evidence. Br J Nutr. 92: 7-19.

[18] Vessby B, et al. (2001) Substituting dietary saturated for monounsaturated fat impairs insulin sensitivity in healthy men and women: The KANWU Study. Diabetologia. 44: 312-9.

[19] Mayer E J, et al. (1993) Usual dietary fat intake and insulin concentrations in healthy women twins. Diabetes Care. 16: 1459-69.

[20] Parker D R, et al. (1993) Relationship of dietary saturated fatty acids and body habitus to serum insulin concentrations: the Normative Aging Study. Am J Clin Nutr. 58: 12936.

[21] Feskens EJ L J, Kromhout D. (1994) Diet and physical activity as determinants of hyperinsulinemia: the Zutphen Elderly Study. Am J Epidemiol. Am J Epidemiol. 140(4): 350-60.

[22] An J, et al. (2004) Hepatic expression of malonyl-CoA decarboxylase reverses muscle, liver and whole-animal insulin resistance. Nat Med. 10: 268-74.

[23] Hulver M W, et al. (2003) Skeletal muscle lipid metabolism with obesity. Am J Physiol Endocrinol Metab. 284: E741-7.

[24] Nagle C A, E L Klett, and R A Coleman (2009) Hepatic triacylglycerol accumulation and insulin resistance. J Lipid Res. 50 Suppl: S74-9.

[25] Vessby B A A, Skarfos E, Berglund L, Salminen I, Lithell H. (1994) The risk to develop NIDDM is related to the fatty acid composition of the serum cholesterol esters. Diabetes. 43: 1353-7.

[26] Erion D M and G I Shulman (2010) Diacylglycerol-mediated insulin resistance. Nat Med. 16: 400-2.

[27] Goodpaster B H and D E Kelley (2002) Skeletal muscle triglyceride: marker or mediator of obesity-induced insulin resistance in type 2 diabetes mellitus? Curr Diab Rep. 2: 21622. 
[28] Holland W L and S A Summers (2008) Sphingolipids, insulin resistance, and metabolic disease: new insights from in vivo manipulation of sphingolipid metabolism. Endocr Rev. 29: 381-402.

[29] Chavez J A and S A Summers (2012) A ceramide-centric view of insulin resistance. Cell Metab. 15: 585-94.

[30] Holland W L, et al. (2007) Inhibition of ceramide synthesis ameliorates glucocorticoid-, saturated-fat-, and obesity-induced insulin resistance. Cell Metab. 5: 167-79.

[31] Wang C-N O B, L., Brindley, D.N (1998) Effects of cell-permeable ceramides and tumor necrosis factor-a on insulin signaling and glucose uptake in 3T3-L1 adipocytes. Diabetes. 47: 24-31.

[32] Hajduch E, et al. (2001) Ceramide impairs the insulin-dependent membrane recruitment of protein kinase B leading to a loss in downstream signalling in L6 skeletal muscle cells. Diabetologia. 44: 173-83.

[33] Bikman BT S S (2011 ) Ceramides as modulators of cellular and whole-body metabolism. J Clin Invest. 121(11): 4222-30.

[34] Glaros E N, et al. (2008) Myriocin slows the progression of established atherosclerotic lesions in apolipoprotein E gene knockout mice. J Lipid Res. 49: 324-31.

[35] Park TS H Y, Noh HL, Drosatos K, Okajima K, Buchanan J, et al. (2008) Ceramide is a cardiotoxin in lipotoxic cardiomyopathy. J Lipid Res. 49(10): 2101-12.

[36] Ussher J R, et al. (2010) Inhibition of de novo ceramide synthesis reverses diet-induced insulin resistance and enhances whole-body oxygen consumption. Diabetes. 59: 245364.

[37] Adams J M, et al. (2004) Ceramide content is increased in skeletal muscle from obese insulin-resistant humans. Diabetes. 53: 25-31.

[38] Schenk S and J F Horowitz (2007) Acute exercise increases triglyceride synthesis in skeletal muscle and prevents fatty acid-induced insulin resistance. J Clin Invest. 117: 1690-8.

[39] Straczkowski M, et al. (2007) Increased skeletal muscle ceramide level in men at risk of developing type 2 diabetes. Diabetologia. 50: 2366-2373.

[40] Dube J J, et al. (2011) Effects of weight loss and exercise on insulin resistance, and intramyocellular triacylglycerol, diacylglycerol and ceramide. Diabetologia. 54: 11471156.

[41] Volek J S, et al. (2009) Carbohydrate Restriction has a More Favorable Impact on the Metabolic Syndrome than a Low Fat Diet. Lipids. 44: 297-309.

[42] Guo Z, et al. (2010) Relationship of the polyunsaturated to saturated fatty acid ratio to cardiovascular risk factors and metabolic syndrome in Japanese: the INTERLIPID study. J Atheroscler Thromb. 17: 777-84.

[43] Hunter J E, J Zhang, and P M Kris-Etherton (2010) Cardiovascular disease risk of dietary stearic acid compared with trans, other saturated, and unsaturated fatty acids: a systematic review. Am J Clin Nutr. 91: 46-63.

[44] Cao H, et al. (2008) Identification of a lipokine, a lipid hormone linking adipose tissue to systemic metabolism. Cell. 134: 933-44. 
[45] Mozaffarian D, et al. (2010) Trans-palmitoleic acid, metabolic risk factors, and newonset diabetes in U.S. adults: a cohort study. Ann Intern Med. 153: 790-9.

[46] Mozaffarian D, R Micha, and S Wallace (2010) Effects on coronary heart disease of increasing polyunsaturated fat in place of saturated fat: a systematic review and metaanalysis of randomized controlled trials. PLoS Med. 7: e1000252.

[47] Jebb S A, et al. (2010) Effect of changing the amount and type of fat and carbohydrate on insulin sensitivity and cardiovascular risk: the RISCK (Reading, Imperial, Surrey, Cambridge, and Kings) trial. Am J Clin Nutr. 92: 748-58.

[48] Astrup A, et al. (2011) The role of reducing intakes of saturated fat in the prevention of cardiovascular disease: where does the evidence stand in 2010? American Journal of Clinical Nutrition. 93: 684-688.

[49] Lee J H, et al. (2009) Omega-3 fatty acids: cardiovascular benefits, sources and sustainability. Nature Reviews Cardiology. 6: 753-758.

[50] Carpentier Y A, L Portois, and W J Malaisse (2006) n-3 fatty acids and the metabolic syndrome. Am J Clin Nutr. 83: 1499S-1504S.

[51] Bang H O, J Dyerberg, and A B Nielsen (1971) Plasma lipid and lipoprotein pattern in Greenlandic West-coast Eskimos. Lancet. 1: 1143-5.

[52] Delarue J, et al. (1996) Effects of fish oil on metabolic responses to oral fructose and glucose loads in healthy humans. Am J Physiol. 270: E353-62.

[53] Weintraub M S, et al. (1988) Dietary polyunsaturated fats of the W-6 and W-3 series reduce postprandial lipoprotein levels. Chronic and acute effects of fat saturation on postprandial lipoprotein metabolism. J Clin Invest. 82: 1884-93.

[54] Woodman R J, et al. (2002) Effects of purified eicosapentaenoic and docosahexaenoic acids on glycemic control, blood pressure, and serum lipids in type 2 diabetic patients with treated hypertension. Am J Clin Nutr. 76: 1007-15.

[55] Mori T A and R J Woodman (2006) The independent effects of eicosapentaenoic acid and docosahexaenoic acid on cardiovascular risk factors in humans. Curr Opin Clin Nutr Metab Care. 9: 95-104.

[56] Delarue J, et al. (2003) Fish oil prevents the adrenal activation elicited by mental stress in healthy men. Diabetes Metab. 29: 289-95.

[57] $\mathrm{Hu} \mathrm{F} \mathrm{B}$, et al. (2002) Fish and omega-3 fatty acid intake and risk of coronary heart disease in women. JAMA. 287: 1815-21.

[58] Connor W E (2000) Importance of n-3 fatty acids in health and disease. Am J Clin Nutr. 71: 171S-5S.

[59] Lombardo Y B and A G Chicco (2006) Effects of dietary polyunsaturated n-3 fatty acids on dyslipidemia and insulin resistance in rodents and humans. A review. J Nutr Biochem. 17: 1-13.

[60] Clarke S D (2001) Polyunsaturated fatty acid regulation of gene transcription: a molecular mechanism to improve the metabolic syndrome. J Nutr. 131: 1129-32.

[61] Jump D B, et al. (2005) Fatty acid regulation of hepatic gene transcription. J Nutr. 135: 2503-6. 
[62] Clamp A G, et al. (1997) The influence of dietary lipids on the composition and membrane fluidity of rat hepatocyte plasma membrane. Lipids. 32: 179-84.

[63] Lombardo Y B, G Hein, and A Chicco (2007) Metabolic syndrome: effects of n-3 PUFAs on a model of dyslipidemia, insulin resistance and adiposity. Lipids. 42: 427-37.

[64] Kopecky J, et al. (2009) n-3 PUFA: bioavailability and modulation of adipose tissue function. Proc Nutr Soc. 68: 361-9.

[65] Neschen S, et al. (2006) Fish oil regulates adiponectin secretion by a peroxisome proliferator-activated receptor-gamma-dependent mechanism in mice. Diabetes. 55: 924-8.

[66] Flachs P, et al. (2005) Polyunsaturated fatty acids of marine origin upregulate mitochondrial biogenesis and induce beta-oxidation in white fat. Diabetologia. 48: 236575.

[67] Huang M, et al. (2012) A high-fat diet enhances proliferation of prostate cancer cells and activates MCP-1/CCR2 signaling. Prostate.

[68] Blank M M, et al. (2012) Dietary fat intake and risk of ovarian cancer in the NIH-AARP Diet and Health Study. Br J Cancer. 106: 596-602.

[69] Rockenbach G, et al. (2011) Dietary intake and oxidative stress in breast cancer: before and after treatments. Nutricion Hospitalaria. 26: 737-744.

[70] Perse M, et al. (2012) High fat mixed lipid diet modifies protective effects of exercise on 1,2 dimethylhydrazine induced colon cancer in rats. Technol Cancer Res Treat. 11: 28999.

[71] Freedland S J, et al. (2008) Carbohydrate restriction, prostate cancer growth, and the insulin-like growth factor axis. Prostate. 68: 11-9.

[72] Lloyd J C, et al. (2010) Effect of isocaloric low fat diet on prostate cancer xenograft progression in a hormone deprivation model. J Urol. 183: 1619-24.

[73] Othman R (2007) Dietary lipids and cancer. Libyan J Med. 2: 180-4.

[74] Bougnoux P, B Giraudeau, and C Couet (2006) Diet, cancer, and the lipidome. Cancer Epidemiol Biomarkers Prev. 15: 416-21.

[75] Suburu J and Y Q Chen (2012) Lipids and prostate cancer. Prostaglandins Other Lipid Mediat. 98: 1-10.

[76] Escrich E, et al. (2011) Modulatory effects and molecular mechanisms of olive oil and other dietary lipids in breast cancer. Curr Pharm Des. 17: 813-30.

[77] Djuric Z (2011) The Mediterranean diet: effects on proteins that mediate fatty acid metabolism in the colon. Nutr Rev. 69: 730-44.

[78] Rose D P and J M Connolly (2000) Regulation of tumor angiogenesis by dietary fatty acids and eicosanoids. Nutr Cancer. 37: 119-27.

[79] Lee J Y, L Zhao, and D H Hwang (2010) Modulation of pattern recognition receptormediated inflammation and risk of chronic diseases by dietary fatty acids. Nutr Rev. 68: 38-61.

[80] Park H, et al. (2011) A high-fat diet increases angiogenesis, solid tumor growth, and lung metastasis of CT26 colon cancer cells in obesity-resistant BALB/c mice. Mol Carcinog. 
[81] Thiebaut A C, et al. (2007) Dietary fat and postmenopausal invasive breast cancer in the National Institutes of Health-AARP Diet and Health Study cohort. J Natl Cancer Inst. 99: 451-62.

[82] Escrich E, et al. (2007) Molecular mechanisms of the effects of olive oil and other dietary lipids on cancer. Mol Nutr Food Res. 51: 1279-92.

[83] Bhatia E, et al. (2011) Chemopreventive effects of dietary canola oil on colon cancer development. Nutr Cancer. 63: 242-7.

[84] Cho K, et al. (2010) Canola oil inhibits breast cancer cell growth in cultures and in vivo and acts synergistically with chemotherapeutic drugs. Lipids. 45: 777-84.

[85] Mamalakis G, et al. (2009) Adipose tissue fatty acids in breast cancer patients versus healthy control women from Crete. Ann Nutr Metab. 54: 275-82.

[86] Mercer N, et al. (2011) Applications of site-specific labeling to study HAMLET, a tumoricidal complex of alpha-lactalbumin and oleic acid. PLoS One. 6: e26093.

[87] Carrillo C, M D Cavia, and S R Alonso-Torre (2011) Oleic acid inhibits store-operated calcium entry in human colorectal adenocarcinoma cells. Eur J Nutr.

[88] Corsetto P A, et al. (2011) Effects of n-3 PUFAs on breast cancer cells through their incorporation in plasma membrane. Lipids Health Dis. 10.

[89] Menendez J A, et al. (2006) HER2 (erbB-2)-targeted effects of the omega-3 polyunsaturated fatty acid, alpha-linolenic acid (ALA; 18:3n-3), in breast cancer cells: the "fat features" of the "Mediterranean diet" as an "anti-HER2 cocktail". Clin Transl Oncol. 8: 812-20.

[90] Berquin I M, et al. (2007) Modulation of prostate cancer genetic risk by omega-3 and omega-6 fatty acids. J Clin Invest. 117: 1866-75.

[91] Doughman S D, S Krupanidhi, and C B Sanjeevi (2007) Omega-3 fatty acids for nutrition and medicine: considering microalgae oil as a vegetarian source of EPA and DHA. Curr Diabetes Rev. 3: 198-203.

[92] Wang D and R N Dubois (2010) Eicosanoids and cancer. Nat Rev Cancer. 10: 181-93.

[93] Larsson S C, et al. (2004) Dietary long-chain n-3 fatty acids for the prevention of cancer: a review of potential mechanisms. Am J Clin Nutr. 79: 935-45.

[94] Nakamura M T, et al. (2004) Mechanisms of regulation of gene expression by fatty acids. Lipids. 39: 1077-83.

[95] Georgiadi A and S Kersten (2012) Mechanisms of gene regulation by fatty acids. Adv Nutr. 3: 127-34.

[96] Swinnen J V, K Brusselmans, and G Verhoeven (2006) Increased lipogenesis in cancer cells: new players, novel targets. Curr Opin Clin Nutr Metab Care. 9: 358-65.

[97] Baracos V E, V C Mazurak, and D W L Ma (2004) n-3 Polyunsaturated fatty acids throughout the cancer trajectory: influence on disease incidence, progression, response to therapy and cancer-associated cachexia. Nutrition Research Reviews. 17: 177-192.

[98] Roberts R, et al. (2009) Markers of de novo lipogenesis in adipose tissue: associations with small adipocytes and insulin sensitivity in humans. Diabetologia. 52: 882-90.

[99] Fernandez-Real J M, et al. (2010) Extracellular fatty acid synthase: a possible surrogate biomarker of insulin resistance. Diabetes. 59: 1506-11. 
[100] Matsuzaka T, et al. (2007) Crucial role of a long-chain fatty acid elongase, Elovl6, in obesity-induced insulin resistance. Nat Med. 13: 1193-202.

[101] Lodhi I J, X C Wei, and C F Semenkovich (2011) Lipoexpediency: de novo lipogenesis as a metabolic signal transmitter. Trends in Endocrinology and Metabolism. 22: 1-8.

[102] Savage D B, et al. (2006) Reversal of diet-induced hepatic steatosis and hepatic insulin resistance by antisense oligonucleotide inhibitors of acetyl-CoA carboxylases 1 and 2. J Clin Invest. 116: 817-24.

[103] Lelliott C and A J Vidal-Puig (2004) Lipotoxicity, an imbalance between lipogenesis de novo and fatty acid oxidation. International Journal of Obesity. 28: S22-S28.

[104] Cases S, et al. (1998) Identification of a gene encoding an acyl CoA:diacylglycerol acyltransferase, a key enzyme in triacylglycerol synthesis. Proc Natl Acad Sci U S A. 95: 13018-23.

[105] Smith S J, et al. (2000) Obesity resistance and multiple mechanisms of triglyceride synthesis in mice lacking Dgat. Nat Genet. 25: 87-90.

[106] Abu-Elheiga L, et al. (2001) Continuous fatty acid oxidation and reduced fat storage in mice lacking acetyl-CoA carboxylase 2. Science. 291: 2613-6.

[107] Donnelly K L, et al. (2005) Sources of fatty acids stored in liver and secreted via lipoproteins in patients with nonalcoholic fatty liver disease. Journal of Clinical Investigation. 115: 1343-1351.

[108] Shimomura I, et al. (1997) Differential expression of exons 1a and 1c in mRNAs for sterol regulatory element binding protein-1 in human and mouse organs and cultured cells. J Clin Invest. 99: 838-45.

[109] Shimano H, et al. (1997) Isoform 1c of sterol regulatory element binding protein is less active than isoform 1a in livers of transgenic mice and in cultured cells. J Clin Invest. 99: 846-54.

[110] Yecies J L, et al. (2011) Akt Stimulates Hepatic SREBP1c and Lipogenesis through Parallel mTORC1-Dependent and Independent Pathways (vol 14, pg 21, 2011). Cell Metab. 14: 280-280.

[111] Yamashita H, et al. (2001) A glucose-responsive transcription factor that regulates carbohydrate metabolism in the liver. Proc Natl Acad Sci U S A. 98: 9116-9121.

[112] lizuka K, et al. (2004) Deficiency of carbohydrate response element-binding protein (ChREBP) reduces lipogenesis as well as glycolysis. Proc Natl Acad Sci U S A. 101: 72817286 .

[113] Ferre P and F Foufelle (2010) Hepatic steatosis: a role for de novo lipogenesis and the transcription factor SREBP-1c. Diabetes Obesity \& Metabolism. 12: 83-92.

[114] lizuka K and Y Horikawa (2008) ChREBP: A glucose-activated transcription factor involved in the development of metabolic syndrome. Endocr J. 55: 617-624.

[115] Dentin R, et al. (2006) Liver-specific inhibition of ChREBP improves hepatic steatosis and insulin resistance in ob/ob mice. Diabetes. 55: 2159-2170.

[116] Verhoeven G (2002) [Androgens and increased lipogenesis in prostate cancer. Cell biologic and clinical perspectives]. Verh K Acad Geneeskd Belg. 64: 189-95; discussion 195-6. 
[117] Mukherjee A, et al. (2012) Lysophosphatidic acid activates lipogenic pathways and de novo lipid synthesis in ovarian cancer cells. J Biol Chem.

[118] Wang G, et al. (2012) Endoplasmic Reticulum Factor ERLIN2 Regulates Cytosolic Lipid Content in Cancer Cells. Biochem J.

[119] Migita T, et al. (2008) ATP citrate lyase: activation and therapeutic implications in nonsmall cell lung cancer. Cancer Res. 68: 8547-54.

[120] Martel P M, et al. (2006) S14 protein in breast cancer cells: direct evidence of regulation by SREBP-1c, superinduction with progestin, and effects on cell growth. Exp Cell Res. 312: $278-88$.

[121] Lin V C, et al. (2012) Activation of AMPK by Pterostilbene Suppresses Lipogenesis and Cell-Cycle Progression in p53 Positive and Negative Human Prostate Cancer Cells. J Agric Food Chem. 60: 6399-407.

[122] Rahman M T, et al. (2012) Fatty acid synthase expression associated with NAC1 is a potential therapeutic target in ovarian clear cell carcinomas. Br J Cancer. 107: 300-7.

[123] Turrado C, et al. (2012) New synthetic inhibitors of Fatty Acid synthase with anticancer activity. J Med Chem. 55: 5013-23.

[124] Jiang B, et al. (2012) Inhibition of Fatty-acid Synthase Suppresses P-AKT and Induces Apoptosis in Bladder Cancer. Urology.

[125] Kuchiba A, et al. (2012) Body mass index and risk of colorectal cancer according to fatty acid synthase expression in the nurses' health study. J Natl Cancer Inst. 104: 41520.

[126] Gelebart P, et al. (2012) Blockade of fatty acid synthase triggers significant apoptosis in mantle cell lymphoma. PLoS One. 7: e33738.

[127] Vazquez-Martin A, et al. (2008) Overexpression of fatty acid synthase gene activates HER1/HER2 tyrosine kinase receptors in human breast epithelial cells. Cell Prolif. 41: 59-85.

[128] Zaytseva Y Y, et al. (2012) Inhibition of fatty acid synthase attenuates CD44-associated signaling and reduces metastasis in colorectal cancer. Cancer Res. 72: 1504-17.

[129] Chen H W, et al. (2012) Targeted therapy with fatty acid synthase inhibitors in a human prostate carcinoma LNCaP/tk-luc-bearing animal model. Prostate Cancer Prostatic Dis.

[130] Yang T P, et al. (2012) Mulberry Leaf Polyphenol Extract Induced Apoptosis Involving Regulation of Adenosine Monophosphate-Activated Protein Kinase/Fatty Acid Synthase in a p53-Negative Hepatocellular Carcinoma Cell. J Agric Food Chem.

[131] Pandey P R, et al. (2012) Anti-cancer drugs targeting fatty acid synthase (FAS). Recent Pat Anticancer Drug Discov. 7: 185-97.

[132] Wang C R S, Watabe K, Liao DF, Cao D. (2010) Acetyl-CoA carboxylase-a as a novel target for cancer therapy. Front Biosci. 2: 515-26.

[133] Zu X Y, et al. (2012) ATP citrate lyase inhibitors as novel cancer therapeutic agents. Recent Pat Anticancer Drug Discov. 7: 154-67. 
[134] Zaidi N, et al. (2012) ATP-citrate lyase (ACLY)-knockdown induces growth arrest and apoptosis through different cell- and environment-dependent mechanisms. Mol Cancer Ther.

[135] Hanai J, et al. (2012) Inhibition of lung cancer growth: ATP citrate lyase knockdown and statin treatment leads to dual blockade of mitogen-activated protein kinase (MAPK) and phosphatidylinositol-3-kinase (PI3K)/AKT pathways. J Cell Physiol. 227: 1709-20.

[136] Wang C, et al. (2009) Acetyl-CoA carboxylase-alpha inhibitor TOFA induces human cancer cell apoptosis. Biochem Biophys Res Commun. 385: 302-6.

[137] Bauer D E, et al. (2005) ATP citrate lyase is an important component of cell growth and transformation. Oncogene. 24: 6314-22.

[138] Yoon S, et al. (2007) Up-regulation of acetyl-CoA carboxylase alpha and fatty acid synthase by human epidermal growth factor receptor 2 at the translational level in breast cancer cells. J Biol Chem. 282: 26122-31.

[139] Luo D X, et al. (2012) Targeting acetyl-CoA carboxylases: small molecular inhibitors and their therapeutic potential. Recent Pat Anticancer Drug Discov. 7: 168-84.

[140] Post J A, J J Bijvelt, and A J Verkleij (1995) Phosphatidylethanolamine and sarcolemmal damage during ischemia or metabolic inhibition of heart myocytes. Am J Physiol. 268: H773-80.

[141] Leonardi R, et al. (2009) Elimination of the CDP-ethanolamine pathway disrupts hepatic lipid homeostasis. J Biol Chem. 284: 27077-89.

[142] Vecchini A, et al. (2000) Molecular defects in sarcolemmal glycerophospholipid subclasses in diabetic cardiomyopathy. J Mol Cell Cardiol. 32: 1061-74.

[143] Lim H Y, et al. (2011) Phospholipid homeostasis regulates lipid metabolism and cardiac function through SREBP signaling in Drosophila. Genes \& Development. 25: 189-200.

[144] Glunde K, C Jie, and Z M Bhujwalla (2004) Molecular causes of the aberrant choline phospholipid metabolism in breast cancer. Cancer Res. 64: 4270-6.

[145] Iorio E, et al. (2005) Alterations of choline phospholipid metabolism in ovarian tumor progression. Cancer Res. 65: 9369-76.

[146] Ross J, et al. (2008) Fatty acid synthase inhibition results in a magnetic resonancedetectable drop in phosphocholine. Mol Cancer Ther. 7: 2556-65.

[147] Lu J, et al. (2002) Role of ether-linked lysophosphatidic acids in ovarian cancer cells. J Lipid Res. 43: 463-76.

[148] Moolenaar W H (1999) Bioactive lysophospholipids and their G protein-coupled receptors. Exp Cell Res. 253: 230-8.

[149] Liscovitch M and L C Cantley (1994) Lipid second messengers. Cell. 77: 329-34.

[150] Westermann A M, et al. (1998) Malignant effusions contain lysophosphatidic acid (LPA)-like activity. Ann Oncol. 9: 437-42.

[151] Freeman M R and K R Solomon (2004) Cholesterol and prostate cancer. J Cell Biochem. 91: 54-69. 
[152] Fang X, et al. (2004) Mechanisms for lysophosphatidic acid-induced cytokine production in ovarian cancer cells. J Biol Chem. 279: 9653-61.

[153] Li H Y, et al. (2003) Cholesterol-modulating agents kill acute myeloid leukemia cells and sensitize them to therapeutics by blocking adaptive cholesterol responses. Blood. 101: 3628-34.

[154] Sivashanmugam P, L Tang, and Y Daaka (2004) Interleukin 6 mediates the lysophosphatidic acid-regulated cross-talk between stromal and epithelial prostate cancer cells. J Biol Chem. 279: 21154-9.

[155] Hu Y L, et al. (2001) Lysophosphatidic acid induction of vascular endothelial growth factor expression in human ovarian cancer cells. J Natl Cancer Inst. 93: 762-8.

[156] Pustilnik T B, et al. (1999) Lysophosphatidic acid induces urokinase secretion by ovarian cancer cells. Clin Cancer Res. 5: 3704-10.

[157] Symowicz J, et al. (2005) Cyclooxygenase-2 functions as a downstream mediator of lysophosphatidic acid to promote aggressive behavior in ovarian carcinoma cells. Cancer Res. 65: 2234-42.

[158] Oyesanya R A, et al. (2008) Transcriptional and post-transcriptional mechanisms for lysophosphatidic acid-induced cyclooxygenase-2 expression in ovarian cancer cells. FASEB J. 22: 2639-51.

[159] Fujita T, et al. (2003) Expression of lysophosphatidic acid receptors and vascular endothelial growth factor mediating lysophosphatidic acid in the development of human ovarian cancer. Cancer Lett. 192: 161-9.

[160] Tanyi J and J Rigo, Jr. (2009) [Lysophosphatidic acid as a potential target for treatment and molecular diagnosis of epithelial ovarian cancers]. Orv Hetil. 150: 1109-18.

[161] Song Y, et al. (2009) Sp-1 and c-Myc mediate lysophosphatidic acid-induced expression of vascular endothelial growth factor in ovarian cancer cells via a hypoxiainducible factor-1-independent mechanism. Clin Cancer Res. 15: 492-501.

[162] Zhao X, et al. (2006) Caspase-3-dependent activation of calcium-independent phospholipase A2 enhances cell migration in non-apoptotic ovarian cancer cells. J Biol Chem. 281: 29357-68.

[163] Song Y, et al. (2007) Inhibition of calcium-independent phospholipase A2 suppresses proliferation and tumorigenicity of ovarian carcinoma cells. Biochem J. 406: 427-36.

[164] McHowat J, et al. (2011) Platelet-activating factor and metastasis: calcium-independent phospholipase A2beta deficiency protects against breast cancer metastasis to the lung. Am J Physiol Cell Physiol. 300: C825-32.

[165] Eder A M, et al. (2000) Constitutive and lysophosphatidic acid (LPA)-induced LPA production: role of phospholipase D and phospholipase A2. Clin Cancer Res. 6: 2482-91.

[166] Shen Y, Y Zheng, and D A Foster (2002) Phospholipase D2 stimulates cell protrusion in v-Src-transformed cells. Biochem Biophys Res Commun. 293: 201-6.

[167] Chae Y C, et al. (2008) Phospholipase D activity regulates integrin-mediated cell spreading and migration by inducing GTP-Rac translocation to the plasma membrane. Mol Biol Cell. 19: 3111-23. 
[168] Kang D W, K Y Choi, and S Min do (2011) Phospholipase D meets Wnt signaling: a new target for cancer therapy. Cancer Res. 71: 293-7.

[169] Ptaszynska M M, et al. (2008) Positive feedback between vascular endothelial growth factor-A and autotaxin in ovarian cancer cells. Mol Cancer Res. 6: 352-63.

[170] Gaetano C G, et al. (2009) Inhibition of autotaxin production or activity blocks lysophosphatidylcholine-induced migration of human breast cancer and melanoma cells. Mol Carcinog. 48: 801-9.

[171] Cowey S and R W Hardy (2006) The metabolic syndrome: A high-risk state for cancer? Am J Pathol. 169: 1505-22.

[172] Pothiwala P, S K Jain, and S Yaturu (2009) Metabolic syndrome and cancer. Metab Syndr Relat Disord. 7: 279-88.

[173] Braun S, K Bitton-Worms, and D LeRoith (2011) The link between the metabolic syndrome and cancer. Int J Biol Sci. 7: 1003-15.

[174] Calle E E, et al. (2003) Overweight, obesity, and mortality from cancer in a prospectively studied cohort of U.S. adults. N Engl J Med. 348: 1625-38.

[175] Emily Jane Gallagher R N, Shoshana Yakar , Derek LeRoith (2010) The Increased Risk of Cancer in Obesity and Type 2 Diabetes: Potential Mechanisms. Principles of Diabetes Mellitus. 579-99. 